\title{
The anticue task: no effect of working memory load on inhibitory control
}

\author{
Nienke Helena Maria van Overveld \\ Faculty of Health, Medicine and Life Sciences \\ nienkevanoverveld@gmail.com
}

\begin{abstract}
Several studies have reported a close link between inhibitory control and working memory (WM), however the exact mechanisms remain elusive. The present study was designed to investigate the effect of WM load on inhibitory control using a novel paradigm - the anticue keypress task. 22 right-handed students ( $21.0 \pm 1.2$ years; 11 males) performed four counterbalanced dual-task conditions on a computer: (pro- / anticue) $\times$ (high / low WM load). Three randomized preparation intervals, i.e. 150, 250 and $450 \mathrm{~ms}$, separated cue and target onsets in all reaction time trials. No interaction was observed for the factor WM load with the pro- or anticue task, $F(1,21)=.21, p=.65$. In conclusion, no distinctive influence of WM load on inhibitory control (anticue) compared to automatic response selection (procue) was found. Therefore, it is suggested that independent neural areas underlie WM performance and inhibitory control.
\end{abstract}

\section{Keywords}

anticue, inhibitory control, reaction time, response selection

\section{Introduction}

Executive functioning, compromising cognitive flexibility, working memory (WM) and inhibitory control, plays a pivotal role in decision making and performing goal-orientated behaviour. In this respect, WM can be seen as a system responsible for the temporary storage, activation and on-line maintenance of information (1). In daily life situations the dynamic environment places high demands on this WM system and performing the intended behaviour becomes challenging. Consequently, a high WM load may lead to a delayed or inappropriate manual response selection. However, the precise interplay between WM and response inhibition remains elusive. Therefore, the present study was designed to investigate the effect of WM load on inhibitory control using a novel behavioural paradigm - the anticue keypress task. 


\section{Anticue task}

The anticue task was based upon the finger-precuing task (Miller, 1982) (2) and established by J. Adam et al. $(3,4)$ as a practical method to measure inhibitory control using reaction time (RT) data. Whereas the original procue task requires an automatic and bottom-up response, the anticue task is cognitive more demanding. In the anticue task, the requested finger-keypress response is located at the contralateral side of the visual cue. First, one must inhibit the automatic ipsilateral response. Second, a volitional component must be initiated, in which the participants must direct their contra-lateral fingers to the target. Therefore, this anticue task can be seen as a proactive and top-down controlled task, which requires time-consuming and effortful processing of the pre-frontal cortex to elicit the correct motor response (5).

Previous research has proposed a critical role for the right inferior frontal cortex in motor inhibition $(6,7)$. More precisely, for proactive selective response suppression, new research evidence has shown that the right inferior frontal cortex seems to interact with the striatum and pallidum (8). Therefore, this basal ganglia's inhibitory indirect pathway is proposed to be activated during the present anticue task.

\section{WM load task}

Simultaneously with the anticue task, a cognitive task can be used to investigate the effect of WM load on inhibitory control. In this study, either one letter (low load) or six letters (high load) had to be remembered. Oi et al. (2014) used this letter recall task to assess the relation between WM load and cognitive control in trait anxiety individuals (9).

The information of a verbal WM load is stored in the articulatory loop of Baddeley's model of WM, which is one of the subsystems of the central 'executive' system (1). Multiple research has shown bilateral activation of the middle frontal gyrus and superior parietal lobule in high verbal WM loads, areas which are not required for low verbal WM load tasks $(10,11)$.

\section{Research question and hypothesis}

The present study was designed to investigate the effect of WM load on inhibitory control using pro- and anticues as a function of preparation interval (PI) (range: 150 - $450 \mathrm{~ms}$ ) in healthy subjects. The different Pls separated cue and target onsets to trace the time course of the interference of WM load with the underlying proactive control process. 
A lower RT (relative to neutral cues) is expected for the procue task since the 4-choice RT task is reduced to a 2-choice RT task. This so-called RT benefit is expected to increase even further when the PI increases to $450 \mathrm{~ms}$, which is the result of increased time of the prefrontal cortex to prepare the correct finger-response (2). As several research has shown no disruptive impact of WM load in pro-saccade tasks $(12,13)$, no significant differences in the procue task were expected. Hence, the automatically activated processes should not be affected by a high cognitive demand.

For the anticue task, RT costs (relative to neutral cues) were expected to emerge for the shortest PI of $150 \mathrm{~ms}$. This time of motor preparation is not sufficient to supress the automatically activated response of the wrong ipsilateral hand via inhibitory control (3-5). In addition, when more WM load is engaged, higher costs and lower benefits are expected for all PIs $(12,13)$. This reflects a less efficient inhibitory control process when WM load increases.

\section{Material and methods}

\section{Participants}

22 Right-handed students (21.0 \pm 1.2 years; 11 males) participated on a voluntary basis. The study was approved by the Ethical Committee of the Faculty of Psychology and Neuroscience of the University of Maastricht. Everybody reported to have normal or normal-to-corrected eyesight and nobody had a history of neurological or psychiatric diseases.

\section{Materials and procedure}

The experiment consisted of a total of four counterbalanced conditions (fig. 1): (pro- / anticue) $\times$ (high / low WM load). The participants were explicitly told what condition to expect and to use the cues to prepare their response. All four conditions were presented in a different block of 120 trials including three randomized PIs (i.e. 150, 250 and $450 \mathrm{~ms}$ ). Half of the trials were neutral cues (4-choice RT task). Each block was divided into $3 \times 40$ trials, with 30 seconds rest. A break of 3 minutes was implemented between each block and each new condition was preceded by 20 practice trials.

Four boxes $(28 \times 28 \mathrm{~mm})$ maintained present overtime on a computer screen, in which red coloured boxes were used for indicating cues and green coloured for indicating the correct finger-keypress response. In the procue task, this cue-target response was located on the ipsilateral side and in the anticue task on the contralateral side. Each response 
was preceded by a small red square for $250 \mathrm{~ms}$. The PI, which separated cue and target onsets, varied randomly between 150, 250 and $450 \mathrm{~ms}$. The appropriate motor response was obtained using the following key allocations: " $Z$ " for left middle finger, " $X$ " for left index finger, "." for right index finger and "I" for right middle finger. After a keypress was obtained, an inter-trial interval of $1.5 \mathrm{~s}$ was used. All participants were instructed to respond as quickly and accurately as possible to the target.

Concurrently, each four consecutive cue-target trials either one letter (low load) or six letters (high load) had to be remembered. This letter (sequence) was present for $6000 \mathrm{~ms}$. A probe was presented to ensure active rehearsal of this letter (sequence). For the correct answer to the probe, the letter ' $\mathrm{F}$ ' was marked in red for 'NO' and the letter ' $\mathrm{K}$ ' in green for 'YES'. To minimize the formation of words, vowels and the letter ' $Y$ ' were excluded from the letter recall task. The probability of the presence of the probe in the letter (sequence) was 0.5 .

Data analysis

Behavioural data with an incorrect keypress response (2.6\%) and responses faster than $150 \mathrm{~ms}$ or in excess of $1000 \mathrm{~ms}$ (0.62\%) were excluded from all statistical analysis (SPSS 15.0). In addition, when an incorrect response was obtained to the letter probe (WM load error), the four RT trials obtained prior to the probe were removed (3.18\%). This was done to comply with the prerequisite to actively load WM with the presented letter (sequence) (9).

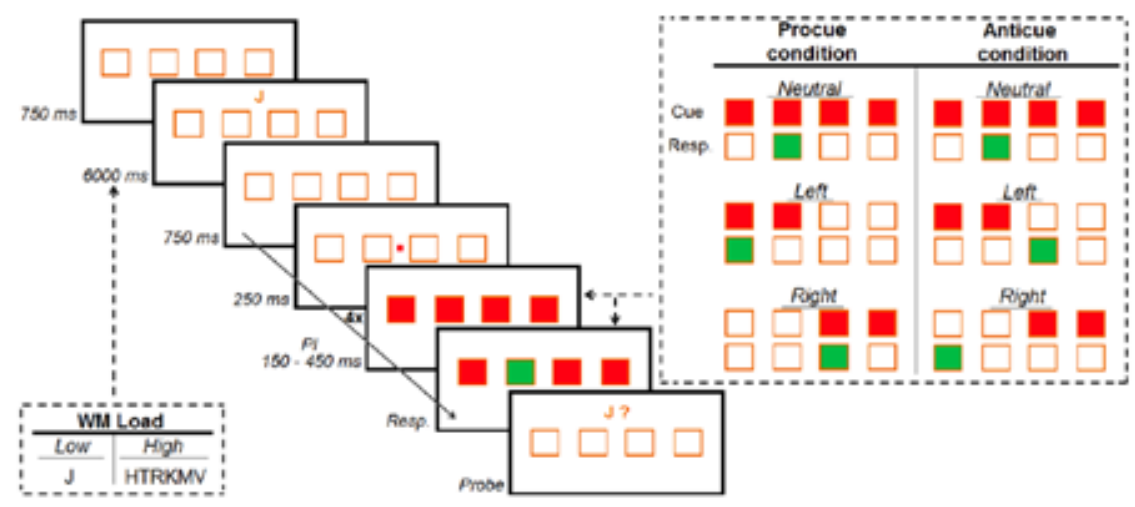

Figure 1. A schematic representation of the response (resp.) display presented in this study. 
The amount of WM load errors was calculated for all four conditions, (pro- / anticue) x (low / high WM load). A repeated-measures analysis of variance (ANOVA) was performed to observe main effects of (and interactions between) the factors block type (pro- / anticue) and WM load (low / high). P-values were obtained from the Huynh-Feldt correction and an alpha-level of .05 was used to assess significant levels.

Another ANOVA was used to investigate the main effects of (and interactions between) the RT data. This analysis used block type (pro- / anticue), WM load (low / high), PI (150, 250 and $450 \mathrm{~ms}$ ) and cue type (neutral / cued) as within-participant factors. To observe main effects of (and interactions between) PI and cue type of the RT data, two additional ANOVAs were performed for the pro- and anticue task. Thereafter, three paired t-test were performed to test significant levels for the RT data: PI x cue type for the pro- and anticue condition.

\section{Results}

\section{WM performance}

The ANOVA yielded a significant main effect of WM load, $F(1,21)=12.00, p<.01$, which showed (fig. 2) that more incorrect responses to the letter probe were made for the high WM load (1.55\%) compared to the low WM load condition (0.36\%). No difference in WM errors between the pro- and anticue condition was found, $F(1,21)=2.90, p=.10$.

\section{Working memory performance}

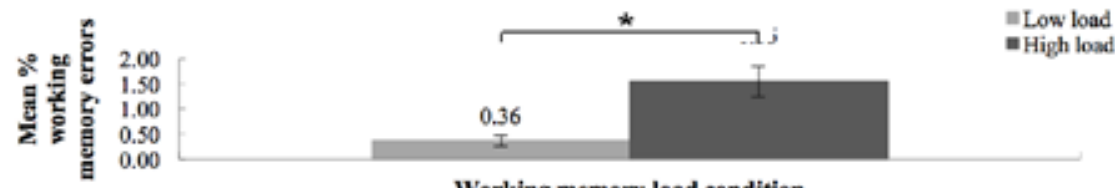

Working memory load condition

Figure 2. Mean \% errors to the letter probe as a function of low / high working memory load, with * $\mathrm{p}<.01$. More errors were made in the high working memory load condition than in the low working memory load condition. Error bars represent standard error.

\section{ANOVA RT data}

The RT data showed significant main effects of block type $(p<.05), \mathrm{PI}(p<.001)$ and cue type $(p<.001)$. These were qualified by a three-way interaction, involving block type, PI and cue type, $F(2,42)=7.22, p<.01$. This complex interaction was decomposed by separate ANOVAs focusing on the procue task and the anticue task. 
In the procue task (fig. 3), the two-way ANOVA reported a significant interaction between $\mathrm{PI}$ and cue type, $\mathrm{F}(2,42)=11.83, \mathrm{p}<.001$. An additional one-sided paired t-test confirmed significant differences between PI and cue type, at all PIs ( $p<.001)$. The RT for both the neutral and cued condition decreased as a function of PI. Overall, this indicates a positive trend towards greater RT benefits when the PI increases, which is shown in figure 5 . This means a faster response is obtained for the cued compared to the neutral condition, with even greater benefits when the PI increases.

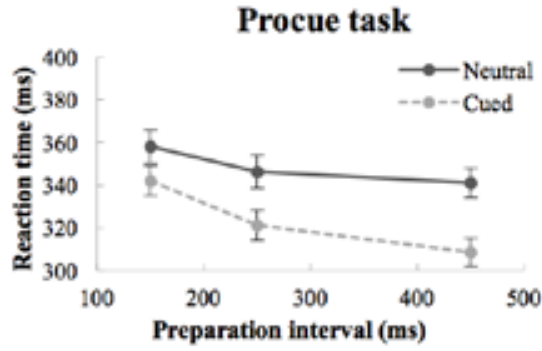

Figure 3. Mean reaction time (ms) of cue type (neutral / cued) for the procue task as a function of preparation interval (150, 250 and $450 \mathrm{~ms})$, with * $p$ <.001. Both the reaction time data of the neutral and cued condition decreased as a function of preparation interval. Error bars represent standard error.

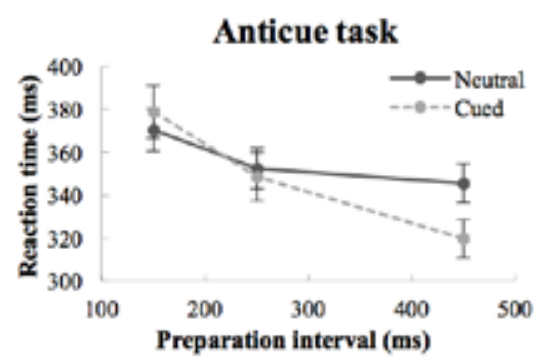

Figure 4. Mean reaction time (ms) of cue type (neutral / cued) for the anticue task as a function of preparation interval (150, 250 and $450 \mathrm{~ms})$, with ${ }^{*} p<.05$ and ${ }^{* *} p<.001$. The reaction time data showed a cross-over pattern (neutral - cued) between the preparation interval of 150 and $250 \mathrm{~ms}$. Error bars represent standard error.

In the anticue task (fig. 4), the two-way ANOVA reported a significant interaction between $\mathrm{PI}$ and cue type, $F(2,42)=29.98, \mathrm{p}<.001$. An additional paired t-test reported a significant difference between $\mathrm{PI}$ and cue type at the PI of 150 and $450 \mathrm{~ms}$, respectively $\mathrm{p}<.05$ and $p<.001$ (one-sided). At the PI of $250 \mathrm{~ms}$, no significant difference was observed ( $p=.36$, two-sided). When the RT cost-benefit pattern was calculated (neutral - cued), a RT cost of $8 \mathrm{~ms}$ was observed at the shortest PI, which is shown in figure 5 . Thereafter, the RT cost became a RT benefit.

Remarkably, no significant main effect was observed for the factor WM load, $F(1,21)=.23$, $p=.64$. In addition, no interaction was observed for the factor WM load with block type, $F(1,21)=.21, p=.65$. This means that neither for the anticue task nor for the procue task, no significant influence of WM load on RT performance was found. 


\section{Discussion/Conclusion}

The present study was designed to investigate the effect of WM load on inhibitory control using pro- and anticues as a function of PI. Herein, the procues reflect automatic response selection, whereas anticues were used to investigate effortful inhibitory control. Therefore, longer RTs were expected to emerge in the anticue condition when the participants were subjected to a high WM load, compared to a low WM load. However, results showed no significant influence of WM load on RT performance, neither in the anticue task nor in the procue task. Therefore, these findings suggest no distinctive influence of WM load on inhibitory control compared to automatic response selection.

\section{Three-way interaction}

Our RT results replicated those of Adam et al. (submitted work), in which both found a significant three-way interaction between block type, $\mathrm{PI}$ and cue type. In addition, Adam et al. used 5 PIs (i.e. 100, 150, 250, 450, 850 ms).

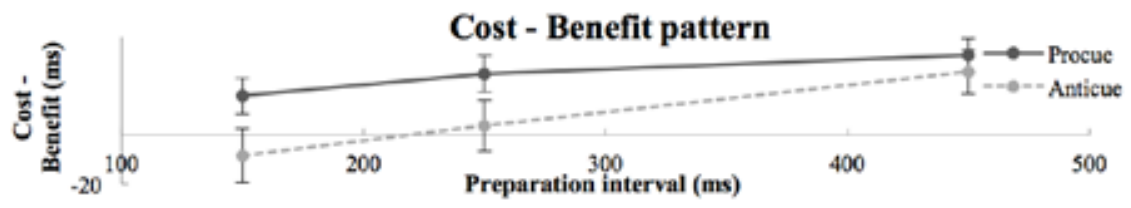

Figure 5. Mean reaction time (ms) cost-benefit pattern (neutral - cued) for the pro- and anticue task as a function of preparation interval (150, 250 and $450 \mathrm{~ms}$ ). The procue condition showed a benefit-only pattern, with greater benefits as the preparation interval increased. The anticue condition showed a cost-benefit pattern. Error bars represent standard error.

Whereas the procue condition reflected an increased RT benefit as a function of Pl, the observed effects for the anticue condition showed a cost-benefit pattern. For the PI of $150 \mathrm{~ms}$, a slower response was obtained for the cued condition compared to the neutral condition. Therefore, the anticue condition confirms the expected effortful inhibition of the pre-potent ipsilateral response (5).

\section{No effect of WM load on inhibitory control process}

Multiple studies have reported an interaction between inhibitory control and WM load, in terms of antisaccade or Go/No-Go task (12 - 14). However, in the present study, no significant interaction between block type (pro- / anticue) and WM load was observed. This contradicts the hypothesis that WM load solely affects the effortful inhibitory control process. 
One possible explanation for this set of discrepant findings is the type of task used to measure inhibitory control. The right inferior frontal cortex is proposed to play a pivotal role in motor inhibition $(6,7)$. However, additional brain area's might be involved in distinctive inhibitory tasks. Therefore, the absence of the effect of the current WM load task on the anticue task can be explained.

\section{Subvocal rehearsal}

While others found an interaction between WM load and inhibitory control, an explanation for our results can be provided by the strategic rehearsal procedure in the high WM load condition of the present letter recall task. The participants implemented an active subvocal rehearsal strategy, which was apparent through silent lip articulation. This was necessary in order to successfully remember six letters, which exceeds the capacity of passive short-term memory (2-3 items) (15).

Subvocal rehearsal is a type of silent inner speech, which is controlled by top-down processes that refresh short-term stored information in the articulatory loop of Baddeley's model of WM (16). Given the role of the left inferior frontal gyrus in language comprehension and production, the left inferior frontal gyrus is proposed to be more activated during high WM load demands (17). In contrast, the right pre-frontal cortex is proposed to play a pivotal role in inhibitory control $(6,7)$. Thus, this current verbal WM load task and the inhibitory control mechanisms can be seen as processes that activate different brain areas. If this is true, then independent neural areas underlie WM performance and inhibitory control, which may explain the absent influence of WM load on inhibitory control using the anticue task in the present study.

\section{Conclusion}

Contrary to the hypothesis, this study showed no significant influence of WM load on RT performance, neither in the anticue task nor in the procue task. Therefore, it is suggested that independent neural areas underlie WM performance and inhibitory control.

\section{Role of the student}

Nienke van Overveld has conducted the research in cooperation with D.A.M.S.W. Frenken and under supervision of J.J. Adam. Data collection and analysis was performed individually. 


\section{References}

1. Baddeley A. Working memory. Science. 1992;255(5044):556-9.

2. Miller J. Discrete versus continuous stage models of human information processing: in search of partial output. J Exp Psychol Hum Percept Perform. 1982;8(2):273-96.

3. Adam JJ, Ament B, Hurks P. Response preparation with anticues in children and adults. J Cogn Psychol. 2011;23(2):264-71.

4. Adam JJ, van Houdt H, Scholtissen B, Visser-Vandewalle V, Winogrodzka A, Duits A. Executive control in Parkinson's disease: effects of dopaminergic medication and deep brain stimulation on anti-cue keypress performance. Neurosci Lett. 2011;500(2):113-7.

5. Ridderinkhof KR. Activation and suppression in conflict tasks: empirical clarification through distributional analyses. Attention Perform. 2002;19:494-519.

6. Aron AR, Robbins TW, Poldrack RA. Inhibition and the right inferior frontal cortex. Trends Cogn Sci. 2004;8(4):170-7.

7. Aron AR, Robbins TW, Poldrack RA. Inhibition and the right inferior frontal cortex: one decade on. Trends Cogn Sci. 2014;18(4):177-85.

8. Majid DS, Cai W, Corey-Bloom J, Aron AR. Proactive selective response suppression is implemented via the basal ganglia. J Neurosci. 2013;33(33):13259-69.

9. Qi S, Zeng Q, Luo Y, Duan H, Ding C, Hu W, et al. Impact of working memory load on cognitive control in trait anxiety: an ERP study. PLoS One. 2014;9(11):e111791.

10. Fegen D, Buchsbaum BR, D'Esposito M. The effect of rehearsal rate and memory load on verbal working memory. Neuroimage. 2015;105:120-31.

11. Barbey AK, Koenigs M, Grafman J. Dorsolateral prefrontal contributions to human working memory. Cortex. 2013;49(5):1195-205.

12. Berggren N, Hutton SB, Derakshan N. The effects of self-report cognitive failures and cognitive load on antisaccade performance. Front Psychol. 2011;2:280.

13. Roberts RJ, Hager LD, Heron C. Prefrontal Cognitive Processes: Working Memory and Inhibition in the Antisaccade Task. J Exp Psychol Gen. 1994;123(4):374-93.

14. Grandjean J, Collette F. Influence of response prepotency strength, general working memory resources, and specific working memory load on the ability to inhibit predominant responses: a comparison of young and elderly participants. Brain Cogn. 2011;77(2):237-47.

15. Waugh NC, Norman DA. Primary Memory. Psychol Rev. 1965;72:89-104.

16. Baddeley A. Working memory: looking back and looking forward. Nat Rev Neurosci. 2003;4(10):829-39.

17. Powell JL, Kemp GJ, Garcia-Finana M. Association between language and spatial laterality and cognitive ability: an fMRI study. Neuroimage. 2012;59(2):1818-29. 